\title{
DOSAGE DE LA GRAISSE DE BEURRE DANS UN MÉLANGE GRAS
}

\author{
par \\ Simonne KUZDZAL-SAVOIE et W. KUZDZAL \\ Station Centrale de Recherches Laitières et de Technologie \\ des Produits Animaux, C.N.R.Z., Jouy-en-Josas (Yvelines)
}

Récemment (Cf. Kuzdzal-Savoie et Kuzdzal, 1968), nous avons décrit une méthode de dosage des acides volatils du beurre, basée sur la différence de solubilité des sels de baryum des acides gras et utilisant la chromatographie en phase gazeuse directe des acides gras.

Cette méthode peut être utilisée en vue de doser la matière grasse de beurre présente dans un mélange gras, puisque l'acide butyrique n'existe en quantité notable que dans la graisse du lait.

Cependant, il convient de vérifier l'efficacité de la méthode dans les cas de mélanges contenant une faible proportion de graisse de beurre. En effet, dans de tels mélanges, les faibles quantités d'acide butyrique qui peuvent provenir des corps gras autres que le beurre, risquent de ne plus être négligeables. Il en résulte une cause d'erreur possible qu'il convient de connaître.

\section{Etude des acides volatils de quelques graisses naturelles}

Nous avons à diverses reprises analysé des échantillons de suif et de saindoux. Sur les chromatogrammes obtenus à partir des acides volatils, on observe des pics correspondant aux acides suivants : propionique, butyrique, hexanoïque, octanoïque. Cependant les proportions de ces acides dans le suif comme dans le saindoux restent faibles (Cf. tableau I).

Par contre la graisse de coprah contient une notable proportion d'acides volatils. Nous avons trouvé $0,005,0,07$ et 0,7 (en g p. $100 \mathrm{~g}$ de graisse) respectivement pour les acides propionique, butyrique et hexanoïque dans un échantillon examiné.

La proportion d'acide octanoïque, trouvée dans cet échantillon, par la méthode aux sels de baryum, est inférieure à la proportion trouvée par l'analyse chromatographique des esters méthyliques totaux. Le dosage de l'acide octanoïque par les sels de baryum 
dans les graisses riches en acide octanoïque (graisse de coprah, graisse de lait de chèvre et de lait de brebis) fera done l'objet d'une étude particulière que nous développerons ultérieurement.

TABLEAU I

ACIDES Volatils

(en $\mathrm{g}$ pour $100 \mathrm{~g}$ de corps gras)

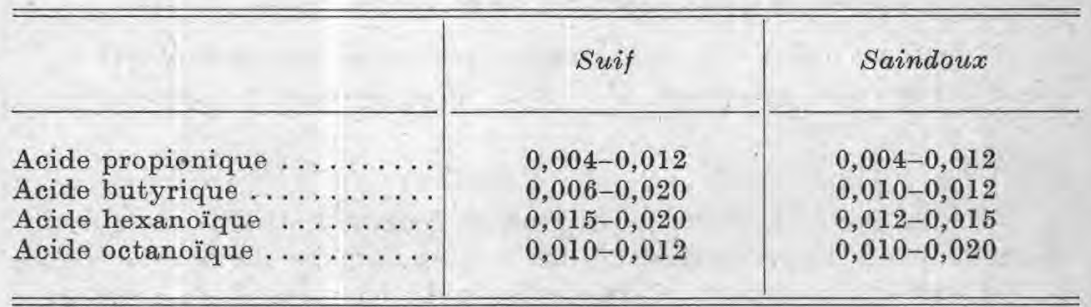

\section{Analyse de mélanges \\ contenant une faible proportion de graisse de beurre}

On a constitué des mélanges contenant de la graisse de suif et de la graisse de beurre. Celle-ci se trouvait dans des proportions variables et connues comprises entre 3 et 7 p. 100. Le beurre utilisé a été l'échantillon no 12/7 fabriqué à Arras (Pas-de-Calais), en octobre 1966 et précédemment analysé (Cf. Kuzdzal-Savoie et Kuzdzal, 1968). Sa teneur en acide butyrique a été estimée à 3 p. $100( \pm 0,2)$. Le suif utilisé eontenait 0,02 p. 100 d'acide butyrique.

Après addition d'une quantité variable d'acide valérique, on a dosé l'acide butyrique présent dans les mélanges en utilisant la technique de dosage des acides volatils par les sels de baryum décrite par ailleurs (Kuzdzal-Savoie et Kuzdzal, 1968).

Dans le tableau II on a groupé les résultats obtenus. On constate que les quantités d'acide butyrique, d'acide hexanoïque et d'acide actanoïque trouvées dans les mélanges s'accroissent en même temps que la proportion de graisse de beurre s'élève.

Mais si l'on considérait que ces acides proviennent exclusivement du beurre, on trouverait pour celui-ci des taux d'acides volatils légèrement supérieurs aux taux antérieurement trouvés.

Du fait de la forte proportion de suif dans les mélanges (93 à 97 p. 100) une quantité d'acide butyrique de l'ordre de $19 \mathrm{mg}$ pour $100 \mathrm{~g}$ de mélange provient du suif.

On a comparé dans le tableau III, les proportions de 'graisse de beurre calculées à partir des résultats du dosage de l'acide butyrique et los proportions connues dans les mélanges de départ. 
TABLEAU II

RÉsultats ANALYTIQUeS

\begin{tabular}{|c|c|c|c|c|c|c|c|c|c|c|c|c|}
\hline \multirow{3}{*}{\multicolumn{3}{|c|}{ Composition des mélanges }} & \multirow{3}{*}{$\begin{array}{c}C_{5} \\
\text { ajouté } \\
(m g)\end{array}$} & \multicolumn{4}{|c|}{$\begin{array}{c}\text { Teneurs en acides volatils * } \\
\qquad(m g)^{\prime}\end{array}$} & \multicolumn{5}{|c|}{ Teneurs en $C_{4} \underset{(\mathrm{mg})}{p .100} \mathrm{~g}$ de mélange } \\
\hline & & & & \multirow[b]{2}{*}{$C_{4}$} & \multirow[b]{2}{*}{$C_{4} \times 1,1$} & \multirow[b]{2}{*}{$C_{6}$} & \multirow[b]{2}{*}{$C_{8}$} & \multirow[b]{2}{*}{ Trouvées } & \multicolumn{3}{|c|}{ Calculées } & \multirow{2}{*}{$\begin{array}{l}\text { Erreur } \\
(\%)\end{array}$} \\
\hline & & & & & & & & & Suif & Beurre & Total & \\
\hline$N^{0} s$. & $\begin{array}{l}\text { Suif } \\
(g)\end{array}$ & $\begin{array}{l}\text { Beurre } \\
(g)\end{array}$ & & & & & & & & & & \\
\hline 1. & 5,000 & 0,158 & 24 & 5,0 & 5,5 & 3,7 & 2,0 & 107 & 19 & 92 & 111 & -4 \\
\hline 2 & 5,000 & 0,214 & 21 & 6,5 & 7,2 & 4,8 & 2,2 & 138 & 19 & 123 & 142 & -3 \\
\hline 3 & 5,000 & 0,256 & 51 & 7,8 & 8,6 & 5,9 & 2,4 & 164 & 19 & 146 & 165 & $-0,6$ \\
\hline 4. & 5,000 & 0,389 & 34 & 12,6 & 13,8 & 8,7 & 3,3 & 256 & 19 & 217 & 236 & +8 \\
\hline
\end{tabular}

* L'analyse porte sur la totalité du mélange préparé. Les résultats sont done exprimés pour ce poids de mélange. 
TABLEAU III

Evaluation de la proportion de gRAisse de BeURRe DANS LE MÉLANGE

\begin{tabular}{|c|c|c|c|c|c|}
\hline & $\begin{array}{l}\text { Co } \\
\text { des }\end{array}$ & & & = & \\
\hline & & $B e$ & o & ntité & ouvée \\
\hline & & & * & ** & $* * *$ \\
\hline $1 \ldots \ldots$ & 96,94 & 3,06 & 2,93 & 3,5 & 3,06 \\
\hline $2 \ldots \ldots$ & 95,90 & 4,10 & 3,97 & 4,6 & 3,93 \\
\hline $3 \ldots \ldots$ & 95,13 & 4,87 & 4,83 & 5,4 & 4,68 \\
\hline $4 \ldots \ldots$ & 92,78 & 7,22 & 7,86 & 8,5 & 7,33 \\
\hline
\end{tabular}

* et ** En se basant sur une teneur en $\mathrm{C}_{4}$ de $3,0 \mathrm{~g}$ pour $100 \mathrm{~g}$ de beurre (beurre $n^{\circ} 12 / 7$ ) et en tenant compte du $C_{4}$ apporté par le suif (*) ou sans en tenir compte $(* *)$.

*** En se basant sur une teneur en $\mathrm{C}_{4}$ de $3,5 \mathrm{~g}$ pour $100 \mathrm{~g}$ de beurre et sans tenir compte du $\mathrm{C}_{4}$ apporté par le suif.

Si l'on tient compte de l'apport en acide butyrique du suif et si l'on se base sur une teneur de 3 p. 100 en acide butyrique du beurre (échantillon $n^{0} 12 / 7$ ), on retrouve les proportions de départ dans des conditions satisfaisantes. Il en est de même si l'on ne tient pas compte de l'acide butyrique provenant du suif et que l'on admette pour le beurre une teneur en acide butyrique de 3,5 p. 100.

Ceci permet d'évaluer l'ordre de grandeur de l'erreur possible. Dans le cas d'un mélange contenant une forte proportion de suif et une faible proportion de beurre, le dosage de l'acide butyrique permet de déterminer la proportion de beurre dans le mélange à 10 p. 100 près, généralement par excès.

Cependant si le mélange contenait une forte proportion, non plus de suif (ou de saindoux), mais de graisse de coprah, le dosage du seul acide butyrique risque de conduire à une erreur plus grande. Dans ce cas les dosages simultanés des acides hexanoïque, butyrique et octanoïque (pour le dernier dans les conditions qui seront ultérieurement précisées) devraient cependant permettre de compléter les informations apportées par le seul dosage de l'acide butyrique.

\section{Conclusion}

Il convient de multiplier les investigations concernant la teneur en acide butyrique ou en un acide possédant le même temps de 
rétention que l'acide butyrique des divers corps gras naturels, afin de connaître les risques d'interférence avec l'acide butyrique du beurre.

Mais d'ores et déjà, le dosage de l'acide butyrique par l'intermédiaire des sels de baryum constitue une méthode satisfaisante en vue du dosage de la graisse de beurre dans un mélange gras.

Reçu pour publication le 27 mai 1968.

\section{Résumé}

Les auteurs proposent d'utiliser le dosage de l'acide butyrique en vue de déterminer la proportion de graisse de beurre dans un mélange gras.

L'acide butyrique est dosé par une technique récemment décrite (cette Revue, 1968), basée sur la différence de solubilité des sels de baryum des acides gras.

\section{Summary}

The proportion of butter fat in a fat mixture is known by the determination of the amount of butyric acid.

For this, the authors use the method described recently (this Revue, 1968) based on the different solubility of the baryum salts of the fatty acids.

\section{RÉFÉRENCE BIBLIOGRAPHIQUE}

Kuzdzal-Savoie (S.) et Kuzdzal (W.), 1968. Utilisation des sels de baryum en vue du dosage des acides gras volatils du beurre. Le Lait, 1968, no 475-76, p. 255 . 